\title{
Examen déontologique des programmes d'immunisation de la santé publique au Canada
}

\author{
Noni E MacDonald ${ }^{1 *}$, Shawn Harmon ${ }^{1,2}$, Janice E Graham ${ }^{1,3}$
}

\section{Résumé}

L'Organisation mondiale de la Santé (OMS) reconnaît que la vaccination est l'une des interventions en matière de santé publique les plus efficaces pour sauver des vies. Lors de l'élaboration d'une feuille de route permettant d'établir un ordre de priorité relatif à I'utilisation des vaccins contre la maladie à coronavirus 2019 (COVID-19) dans un contexte d'approvisionnement limité, l'OMS a souligné l'importance d'un cadre de valeurs (principes éthiques). La vaccination doit faire l'objet d'un examen éthique indépendant des données de recherche sur les vaccins, des pratiques de fabrication, de l'assurance juridique et éthique du consentement éclairé, ainsi que des questions de justice sociale concernant l'équité des programmes, y compris le droit d'accès. Un examen déontologique du programme de vaccination de l'Australie a été rapporté en 2012. Ce dossier CANVax (Centre canadien de ressources et d'échange sur les données probantes en vaccination) propose un examen déontologique de la vaccination au Canada en utilisant les critères utilisés pour l'Australie.
Cette oeuvre est mise à la disposition selon les termes de la licence internationale Creative Commons Attribution 4.0

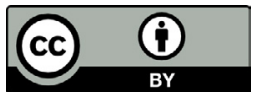

Affiliations

${ }^{1}$ Département de pédiatrie, Université Dalhousie, IWK Health Centre, Halifax, NS

${ }^{2}$ Analyste des politiques, Health Law Institute, Schulich School of Law, Université Dalhousie, Halifax, NS

${ }^{3}$ Unité de recherche en technoscience et réglementation, Faculté de médecine, Université Dalhousie, Halifax, NS

\section{*Correspondance :}

noni.macdonald@dal.ca

Citation proposée : MacDonald NE, Harmon S, Graham JE. Examen déontologique des programmes d'immunisation de la santé publique au Canada. Relevé des maladies transmissibles au Canada 2021;47(4):259-64. https://doi.org/10.14745/ccdr.v47i04a09f

Mots-clés : éthique, immunisation, santé publique, droit de la santé, Canada

\section{Introduction}

L'Organisation mondiale de la Santé (OMS) reconnaît que la vaccination est l'une des interventions de santé publique les plus efficaces pour sauver des vies (1). Les effets de la vaccination vont bien au-delà de la santé et du bien-être des personnes et des communautés, car ils améliorent les déterminants sociaux de la santé qui ont une incidence sur la productivité en milieu de travail, l'équité, la stabilité institutionnelle, le développement économique et l'innovation (voir figure 1). La pandémie de maladie à coronavirus 2019 (COVID-19) a attiré l'attention du monde entier sur l'importance des interventions de santé publique et de la vaccination pour rétablir la santé et le bien-être des personnes et des communautés. En élaborant une feuille de route qui permet d'établir un ordre de priorité relatif à l'utilisation des vaccins contre la COVID-19 dans un contexte d'approvisionnement limité (2), l'OMS a souligné l'importance d'un cadre de valeurs (3). Ce cadre de valeurs s'appuie sur des principes déontologiques axés sur le bien-être humain, le respect égal, l'équité mondiale, l'équité nationale, la réciprocité et la légitimité. Ces principes, bien qu'importants pour les vaccins contre la COVID-19, s'appliquent à tous les programmes de vaccination. La vaccination doit être assujettie à des règles d'éthique indépendantes concernant les données de la recherche sur les vaccins et des pratiques de fabrication, de l'assurance juridique et éthique du consentement éclairé, et des questions de justice sociale, notamment le droit d'accès.
Figure 1 : La vaccination et les objectifs de développement durable

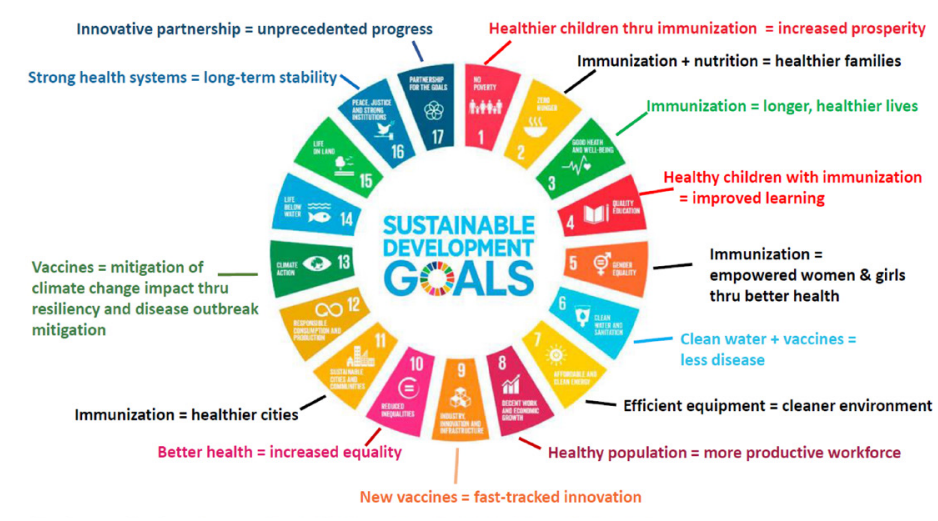

Adapté de GAVI - I'Alliance du Vaccin (https://www.gavi.org/fr/sante-mondiale-etdeveloppement/objectifs-de-developpement-durable)

(Figure en anglais seulement)

Dans ce dossier, nous proposons un "bilan à des fins éthiques " des programmes d'immunisation de la santé publique au Canada et suggérons des améliorations à apporter. 


\section{Les critères de contrôle utilisés}

Les programmes de vaccination méritent d'être examinés sous un angle déontologique, non seulement par les praticiens qui administrent les vaccins aux patients, mais aussi pour s'assurer que les principes déontologiques sont respectés dans l'ensemble du programme, car les communautés et les populations visées par la vaccination varient en fonction de l'âge, du fardeau de la maladie, de la vulnérabilité et de la marginalisation. Les risques et les avantages de la vaccination peuvent varier dans les communautés considérées comme étant vulnérables (e.g. les nourrissons, les enfants, les femmes enceintes, les personnes immunodéprimées et les personnes âgées).

L'attention portée aux questions d'éthique s'est principalement concentrée sur les risques et avantages nationaux aux fins de recommandations politiques, ainsi que sur la confiance sous l'angle de l'hésitation et l'acceptation des vaccins, tandis que les questions d'éthique qui se posent en première ligne (avantages, risques, efficacité, équité et justice, autonomie, réciprocité et confiance) ont reçu moins d'attention. L'évaluation éthique des programmes de vaccination à l'échelle nationale est un domaine relativement nouveau.

Isaacs (4) a élaboré sept principes éthiques pour éclairer les évaluations des programmes de vaccination de la santé publique en Australie. Ils s'appliquent à l'ensemble du programme, de la politique aux pratiques de première ligne:

- Avantages

- Risques

- Efficacité

- Équité et justice

- Autonomie

- Réciprocité

- Confiance

Ce dossier CANVax (Centre canadien de ressources et d'échange sur les données probantes en vaccination) applique les critères éthiques australiens d'Isaac (4) au Canada et attire l'attention sur plusieurs préoccupations.

\section{Application des critères}

Au niveau des politiques, le Comité consultatif national de I'immunisation (CCNI), chacune des provinces et chacun des territoires décident qui est admissible ou non aux vaccins en se fondant sur des données probantes. Le CCNI s'efforce d'intégrer l'éthique, l'équité, la faisabilité et l'acceptabilité dans ses recommandations nationales (5). L'évaluation des résultats de l'application de ces principes d'éthique ne sera connue qu'avec le temps.

Comme il a été mentionné, le CCNI formule des recommandations fondées sur des données probantes qui tiennent compte du fardeau de la population et des risques de la maladie, y compris l'innocuité et les avantages prévus du vaccin dans différentes populations au Canada. Les provinces et les territoires prennent ensuite leurs décisions en matière de politique de santé publique quant à l'inclusion et au paiement du ou des vaccins dans leurs programmes d'immunisation, aux personnes à qui ils seront offerts, à la façon dont le programme sera exécuté et à l'introduction éventuelle de programmes de rattrapage. (Cela n'inclut pas les vaccins contre la COVID-19, que le gouvernement fédéral prend en charge.)

Les vaccins recommandés par le CCNI ne sont pas tous également accessibles dans l'ensemble du pays. Les différences provinciales dans la mise en œuvre peuvent entraîner la confusion chez les prestataires et le public, ce qui soulève des problèmes d'équité nationale et de justice sociale en matière d'accès. Par exemple, l'introduction du vaccin contre le pneumocoque à protéines conjuguées et du vaccin contre la varicelle varie d'un pays à l'autre, bien que ces deux maladies pouvant être prévenues par la vaccination soient répandues partout (6). La disparité dans le calendrier d'introduction des vaccins se poursuit, l'Alberta ayant introduit un programme de vaccination contre le virus du papillome humain (VPH) en milieu scolaire pour les filles en 2008 et le Nunavut ne l'ayant fait qu'en 2010. Les provinces et territoires ne proposent pas tous des programmes de rattrapage du vaccin contre le VPH pour les garçons, et l'approbation par la Nouvelle-Écosse du vaccin systématique contre le rotavirus dans son programme pour les nourrissons n'est intervenue qu'en 2019, des années après les autres provinces (7). Ainsi, l'accès aux vaccins recommandés par le CCNI varie selon l'endroit où l'on vit au Canada, ce qui met en évidence les questions d'équité et de justice sociale.

\section{Risque, avantages et efficacité}

Au Canada, les systèmes de surveillance permettent de suivre à la fois les avantages de l'immunisation, i.e. la prévalence de la maladie pouvant être prévenue par la vaccination, et les risques de l'immunisation, i.e. les manifestations cliniques indésirables à la suite de l'immunisation. Le Canada dispose d'un système de déclaration passive et active des manifestations cliniques indésirables à la suite de la vaccination (8) et de la maladie pouvant être prévenue par la vaccination, la déclaration de cette dernière par les laboratoires étant désormais plus automatisée que par le passé. Les résultats des résumés des maladies pouvant être prévenues par la vaccination et des manifestations cliniques indésirables à la suite de la vaccination sont régulièrement examinés et discutés dans les provinces ou territoires où ils sont signalés, ainsi qu'à l'échelle nationale au CCNI et à l'Agence de la santé publique du Canada (l'Agence). Il existe de nombreux exemples où l'introduction d'un nouveau vaccin au Canada a entraîné une diminution marquée de la maladie pouvant être prévenue par la vaccination, par exemple pour les maladies invasives à Haemophilus influenzae de type b (9) et les maladies liées au pneumocoque (10), ce qui fournit des données sur l'efficacité des vaccins dans les populations canadiennes. IMPACT (programme canadien de surveillance active de l'immunisation), le réseau national canadien de surveillance 
active en milieu hospitalier pédiatrique, qui recherche les échecs vaccinaux, les manifestations cliniques indésirables graves à la suite de la vaccination (i.e. les hospitalisations) et certaines maladies pouvant être prévenues par la vaccination et maladies infectieuses, a fourni de nombreuses données précieuses sur les effets des vaccins pédiatriques au cours des deux dernières décennies. Des données similaires concernant les effets des vaccins pour adultes ne sont pas recueillies de manière systématique. Le réseau SOS (Serious Outcomes Surveillance) recueille des données sur les adultes admis à I'hôpital en raison d'une grippe ou d'une pneumonie, mais il ne suit pas les manifestations cliniques indésirables graves à la suite de la vaccination (11). Aucun de ces systèmes ne recueille de données sur l'utilisation des vaccins dans la région spécifique desservie par le site de déclaration, ce qui signifie que l'efficacité des différents vaccins au niveau local ne peut être qu'estimée. L'application CANImmunize adaptée aux vaccins contre la COVID-19 comble certaines de ces lacunes, mais n'est pas entièrement utilisée dans tout le pays (12).

Les échecs vaccinaux sont moins bien suivis. Le signalement par les laboratoires de microbiologie des maladies causées par un microbe pouvant être prévenues par la vaccination est de plus en plus actif dans tout le pays à mesure qu'ils s'automatisent. Cependant, les cas de maladies pouvant être prévenues par la vaccination détectés peuvent manquer d'informations pour savoir s'il s'agit d'un échec vaccinal. La nécessité de détecter l'échec de vaccins contre la COVID-19 - dus à l'échec du vaccin lui-même et à des erreurs de programme telles qu'une mauvaise manipulation de l'entreposage du vaccin et des populations ou sous-groupes manqués - afin d'établir l'efficacité dans différents groupes d'âge et contextes a poussé toutes les provinces et tous les territoires à recueillir des données d'immunisation pour ces vaccins afin de pouvoir détecter plus facilement les échecs de réception du vaccin ainsi que les échecs d'immunisation. Cependant, toutes les régions ne disposent pas de registres d'immunisation de qualité pour tous les vaccins. Dans l'ensemble, les principes de risque et d'efficacité pourraient être plus largement et mieux traités dans tous les groupes d'âge.

\section{Autonomie et consentement éclairé}

En ce qui concerne l'autonomie, les programmes de vaccination en milieu scolaire soulèvent des questions non résolues de consentement. Ces programmes sont couramment offerts au Canada, mais l'âge varie, de la 4e année (environ 10-11 ans) à la $9^{e}$ année (environ 15-16 ans). En général, les parents signent un formulaire de consentement pour l'administration des vaccins, mais s'il n'y a pas de consentement et que l'élève veut être vacciné, ou si le consentement et la décision déclarée de l'élève sont en désaccord, les praticiens de la santé publique peuvent ne pas savoir comment procéder et les pratiques des différentes juridictions diffèrent (13). Dans le cas d'un consentement parental positif avec un refus de l'élève, il est peu probable que la vaccination ait lieu. Mais pour l'élève qui exprime le désir de se faire vacciner face au refus parental, il y a plus d'ambiguïté. Cette incertitude est déconcertante en raison des variations, à travers le Canada, de l'âge auquel la " capacité » est supposée, et des différences d'approche des « mineurs matures » (14). En Ontario, par exemple, il n'y a pas d'âge précis auquel la capacité d'un mineur est présumée; les enfants sont évalués sur leur capacité à donner leur consentement, et cela dépend moins de leur âge que de leur compréhension, que le prestataire de soins doit évaluer (15). En outre, certains travailleurs de la santé ne passeront pas outre le veto parental sur la vaccination, même si le mineur semble avoir toutes ses capacités. Une norme nationale de pratique n'a pas encore été établie, ce qui peut empiéter sur les droits des adolescents et miner les normes de consentement éthique.

Une autre incertitude liée au consentement éclairé concerne l'information sur les vaccins qui répond aux besoins des utilisateurs. Au Canada, chaque province et territoire élabore ses propres dossiers d'information à l'intention des patients et des parents. Bien qu'ils puissent s'inspirer des informations et des conseils du CCNI, ils diffèrent souvent, ce qui laisse planer une incertitude quant à la description du même vaccin (16). Pour ceux qui cherchent à prendre une décision éclairée, cela peut être déroutant, voire rebutant, surtout lorsque les antivaccinalistes (" antivaxxers ») déclarent que les informations fournies sont inadéquates. Une meilleure collaboration dans l'élaboration de documents d'information sur les vaccins pourrait rendre ce domaine moins confus et moins litigieux pour ceux qui cherchent des informations, et elle pourrait améliorer la couverture dans les populations où de nouvelles données confirment la sécurité et l'efficacité.

\section{Réciprocité}

Le Canada présente un tableau mitigé concernant la réciprocité pour les programmes d'immunisation de santé publique. Les coûts des soins hospitaliers associés à des manifestations cliniques indésirables rares et graves à la suite de la vaccination sont pris en charge par le système de santé public. Toute invalidité à long terme causée par une manifestation clinique indésirable à la vaccination peut être prise en charge, en partie, par les prestations d'invalidité offertes par les ministères des services sociaux et communautaires des provinces et des territoires. Toutefois, ces prestations ne couvrent généralement pas la perte de revenu à vie due à l'incapacité de travailler. Mais la causalité du vaccin n'est pas toujours facile à évaluer. Par exemple, l'évaluation de la causalité des incidents de narcolepsie en Finlande et en Suède après l'administration du vaccin H1N1 est confondue par le risque accru connu de narcolepsie avec l'infection $\mathrm{H} 1 \mathrm{~N} 1$, le virus circulant déjà dans la communauté lorsque les programmes de vaccination ont commencé (17). II n'est pas simple de démêler l'effet de l'infection naturelle concomitante de l'immunisation. L'apparition de la narcolepsie dans l'enfance a un effet profond sur le niveau d'éducation, la capacité de travail et de nombreux résultats sociaux, familiaux 
et personnels. De solides arguments éthiques plaident en faveur d'un dédommagement pour des manifestations cliniques indésirables aussi graves à la suite de la vaccination, lorsqu'elles sont dues à l'immunisation, en particulier si les vaccins sont obligatoires (18). Au Canada, seul le Québec dispose d'un programme d'indemnisation en cas de préjudice lié aux vaccins, en place depuis 1987 (19). En décembre 2020, le Canada a annoncé que l'Agence mettait en œuvre un programme de soutien aux préjudices involontaires liés aux vaccins pour tous les vaccins approuvés par Santé Canada (20), mais les détails sont en attente en avril 2021. Il s'agit d'une entreprise complexe : il existe sept caractéristiques essentielles pour assurer le fonctionnement équitable, transparent et efficace d'un programme d'indemnisation des victimes de vaccins (21).

\section{Confiance, justice sociale et équité}

Les problèmes de confiance peuvent se poser pour les programmes publics de vaccination dans un certain nombre de domaines différents. Un ensemble de manifestations cliniques indésirables à la suite de la vaccination peut miner la confiance dans le programme et dans le vaccin si leur causalité n'est pas traitée rapidement et de manière satisfaisante. Cela s'est produit au Japon et au Danemark avec le vaccin contre le papillome humain $(22,23)$. II n'est pas clair si toutes les provinces et tous les territoires ou l'Agence ont mis en place des procédures d'examen scientifique rapide et des modèles de communication sérieux en matière de manifestations cliniques indésirables à la suite de la vaccination, ou si la coordination entre les niveaux de gouvernement dans le pays est bonne. II importe de faire remarquer que la dernière mise à jour semestrielle signalée du Système canadien de surveillance des effets secondaires suivant I'immunisation (SCSESSI) porte sur la période de janvier à juin 2019 (24). Bien que la pandémie de COVID-19 ait pu retarder les mises à jour, il est inquiétant qu'aucun nouveau rapport de synthèse n'ait été disponible pour les manifestations cliniques indésirables à la suite de la vaccination au cours des 18 derniers mois.

La communication de messages contradictoires sur les manifestations cliniques indésirables à la suite de la vaccination, qu'ils soient liés au vaccin ou une coïncidence, peut rapidement saper la confiance du public (25). L'attention des médias lorsque la Norvège a constaté des décès chez des personnes âgées fragiles à la suite de la vaccination par la COVID-19 illustre à quel point la confiance peut être facilement ébranlée (26).

Des problèmes de confiance se posent également lorsque différents vaccins sont disponibles pour la même maladie, mais que certains sont financés et d'autres non (e.g. différents vaccins contre la grippe destinés aux personnes âgées) (27). Cela soulève également des questions d'éthique concernant l'évaluation du rapport coût-efficacité, la justice sociale et l'évaluation de l'équité. La contribution du Canada à COVAX, le partage mondial des vaccins contre la COVID-19, puis le projet du Canada de recevoir des vaccins de COVAX, contrairement à de nombreux autres pays à revenu élevé, ont soulevé des inquiétudes quant à l'engagement du Canada envers l'équité mondiale en matière de vaccins (28). Là encore, la communication est essentielle. Nous avons besoin de discussions sur les valeurs et les priorités publiques qui incluent les voix de diverses communautés. En outre, il faut montrer que ces voix sont appréciées et prises en considération, faute de quoi la confiance sera ébranlée.

\section{Considérations éthiques pour les professionnels de la santé qui pratiquent la vaccination}

Les infirmières de santé publique, les médecins et leur personnel de bureau représentent la première ligne d'interaction avec les patients et les soignants dans les situations de vaccination. Code d'éthique et de professionnalisme de l'Association médicale canadienne (29) et le Code de déontologie des infirmières et infirmiers autorisés de l'Association des infirmières et infirmiers du Canada (30) énoncent les engagements et responsabilités éthiques et professionnels des professions médicales et infirmières. Les codes fournissent des normes de pratique éthique pour guider les médecins et les infirmières dans l'accomplissement de leur obligation de fournir le plus haut niveau de soins, et pour favoriser la confiance des patients et du public dans les professions médicales.

Les codes ne sont pas exhaustifs; ils visent à fournir des normes de pratique éthique qui peuvent être interprétées et appliquées dans des situations particulières. La manière dont ils pourraient être appliqués aux situations de vaccination, en particulier pour les vaccins contre la COVID-19 en ces temps de contraintes vaccinales, n'a pas été bien articulée.

\section{Conclusion}

Dans l'ensemble, ce bilan éthique de haut niveau relatif aux programmes canadiens d'immunisation en santé publique suggère que le Canada est généralement sur la bonne voie, même s'il y a place à l'amélioration. Le Canada a obtenu la "note de passage » en ce qui concerne des principes tels que les avantages, le risque et l'efficacité. Il existe cependant des lacunes et des incertitudes concernant d'autres principes (i.e. l'équité, la justice, l'autonomie, la réciprocité, la confiance). Celles-ci témoignent de la nécessité d'accorder une plus grande attention aux questions touchant à d'autres principes, notamment la solidarité et la transparence. Le contexte de contrainte du vaccin contre la COVID-19 a encore exacerbé certaines de ces préoccupations.

II n'est pas facile de trouver des solutions universelles, étant donné la taille du Canada et la structure de son système de santé; les différences régionales dans la prévalence et le fardeau des maladies pouvant être prévenues par la vaccination 
entraînent des décisions différentes. Les priorités provinciales et territoriales en matière de soins de santé entraînent également des variations dans la mise en œuvre. Ayant reconnu cela, des mesures facilement justifiables qui peuvent améliorer la cohérence collective dans tous les milieux de l'immunisation au Canada peuvent être mises en place à court terme; la fragmentation et la duplication sont problématiques. Par exemple, en fournissant un simple questionnaire d'auto-évaluation de l'éthique aux provinces et aux territoires, on pourrait déterminer les défis communs et propres à chaque région et stimuler l'élaboration d'outils et de réponses communs efficaces. En outre, l'élaboration conjointe de pratiques exemplaires pour des questions telles que le consentement dans les programmes de vaccination en milieu scolaire aurait des avantages à l'échelle du pays. Enfin, un examen complet des manifestations cliniques indésirables graves à la suite de la vaccination et l'élaboration d'un modèle de communication en cas de crise due aux manifestations cliniques indésirables à la suite de la vaccination sont indéniablement utiles et relèvent des capacités existantes. La distribution en masse du vaccin contre la COVID-19 a accentué la pression sur ce point. Un leadership est nécessaire pour relever ces défis afin d'améliorer les résultats du prochain contrôle déontologique, qui pourrait prendre en compte la solidarité et la transparence.

\section{Déclaration des auteurs}

N. E. M. - Conceptualisation, rédaction du projet original S. H. - Conceptualisation, révision des projets, accord sur le projet final

J. E. G. - Conceptualisation, révision des projets, accord sur le projet final

\section{Intérêts concurrents}

Aucun.

\section{Financement}

Aucun financement externe.

\section{Références}

1. Strategic Advisory Group of Experts on Immunization. Immunization today and in the next decade: 2018 Assessment report of the Global Vaccine Action Plan. Geneva: World Health Organization; 2018. https://www.who. int/immunization/global_vaccine_action_plan/SAGE_GVAP_ Assessment_Report_2018_EN.pdf?ua=1
2. SAGE Working Group on COVID-19 Vaccines. WHO sage roadmap for prioritizing uses of COVID-19 vaccines in the context of limited supply: an approach to inform planning and subsequent recommendations based upon epidemiologic setting and vaccine supply scenarios. Version 1.1. Geneva: World Health Organization; 2020. https:// www.who.int/docs/default-source/immunization/sage/covid/ sage-prioritization-roadmap-covid19-vaccines.pdf?Status $=\mathrm{Te}$ mp\&sfvrsn=bf227443_2

3. SAGE Working Group on COVID-19 Vaccines. WHO SAGE values framework for the allocation and prioritization of COVID-19 vaccination. Geneva: World Health Organization; 2020. https://apps.who.int/iris/ bitstream/handle/10665/334299/WHO-2019-nCoV-SAGE_ Framework-Allocation_and_prioritization-2020.1-eng. pdf?ua $=1$

4. Isaacs D. An ethical framework for public health immunisation programs. N S W Public Health Bull 2012;23(5-6):111-5. DOI PubMed

5. Comité consultatif national de l'immunisation (CCNI). Comité consultatif national de l'immunisation (CCNI) : Plan de travail pour 2019-2020. Ottawa (ON) : Gouvernement du Canada; (modifié 2020-04-22; accédé 2021-03-31). https:// www.canada.ca/en/public-health/services/immunization/ national-advisory-committee-on-immunization-naci/ workplan.html

6. Halperin SA, Pianosi K. Immunization in Canada: a 6-year update. J Can Chiropr Assoc 2010;54(2):85-91. PubMed

7. Programmes de vaccination systématique et de rattrapage des provinces et des territoires pour les nourrissons et les enfants au Canada. Ottawa (ON) : Gouvernement du Canada; (modifié 2020-12-11; accédé 2021-03-21). https://www.canada.ca/fr/sante-publique/ services/renseignements-immunisation-provinces-etterritoires/programmes-vaccination-systematique-provin ces-territoires-nourrissons-enfants.html

8. MacDonald NE, Law BJ. Canada's eight-component vaccine safety system: A primer for health care workers. Paediatr Child Health 2017;22(4):e13-6. DOI PubMed

9. Scheifele DW. Recent trends in pediatric Haemophilus influenzae type B infections in Canada. Immunization Monitoring Program, Active (IMPACT) of the Canadian Paediatric Society and the Laboratory Centre for Disease Control. CMAJ 1996;154(7):1041-7. PubMed

10. Kellner J; Canadian Paediatric Society, Infectious Diseases and Immunization Committee. Update on the success of the pneumococcal conjugate vaccine. Paediatr Child Health 2011;16(4):233-40. DOI PubMed

11. Canadian Immunization Research Network(CIRN). Serious Outcomes Surveillance (SOS) Network: what is SOS. Halifax (NS): Canadian Immunization Research Network; (accédé 2021-03-21). http://cirnetwork.ca/network/ serious-outcomes/

12. CANImmunize. Un dossier de vaccination électronique pour les Canadiens. Ottawa (ON) : CANimmunize; (accédé 2021-04-15). https://www.canimmunize.ca/fr/home 
13. Glauser W. Teens, vaccines and the age of consent. CMAJ 2019;191(12):E348-9. DOI PubMed

14. Coughlin KW; Canadian Paediatric Society Committee on Bioethics. Medical decision-making in paediatrics: infancy to adolescence. Paediatr Child Health 2018;23(2):138-46. DOI PubMed

15. Schwartz Y, Williams TS, Roberts SD, Hellmann J, Zlotnik Shaul R. Adolescent decision-making in Canadian medical contexts: integrating neuroscience and consent frameworks. Paediatr Child Health 2018;23(6):374-6. DOI PubMed

16. Steenbeek A, Macdonald N, Downie J, Appleton M, Baylis F. Ill-informed consent? A content analysis of physical risk disclosure in school-based HPV vaccine programs. Public Health Nurs 2012;29(1):71-9. DOI PubMed

17. Sarkanen TO, Alakuijala AP, Dauvilliers YA, Partinen MM. Incidence of narcolepsy after H1N1 influenza and vaccinations: systematic review and meta-analysis. Sleep Med Rev 2018;38:177-86. DOI PubMed

18. Attwell K, Drislane S, Leask J. Mandatory vaccination and no fault vaccine injury compensation schemes: an identification of country-level policies. Vaccine 2019;37(21):2843-8. DOI PubMed

19. Gouvernement du Québec. Programme d'indemnisation des victimes d'une vaccination. Québec (OC) : Gouvernement du Québec; (modifié 2021-03-17; accédé 2021-03-31). https:// www.quebec.ca/sante/conseils-et-prevention/vaccination/ indemnisation-des-victimes-vaccination

20. Agence de la santé publique du Canada. Le gouvernement du Canada annonce la mise sur pied du programme pancanadien de soutien aux victimes d'une vaccination. Communiqué de presse. 10 décembre 2020 - Ottawa (ON) : ASPC; (accédé 2021-03-31). https://www. canada.ca/fr/sante-publique/nouvelles/2020/12/ le-gouvernement-du-canada-annonce-la-mise-sur-pi ed-du-programme-pancanadien-de-soutien-aux-vic times-dune-vaccination.html

21. Harmon $\mathrm{SH}$. Characteristics of a fair vaccine injury compensation program for Canada. RSC COVID-19 Series. Publication \#87. Ottawa (ON): Royal Society of Canada; 2021 (accédé 2021-04-15). https://rsc-src.ca/en/voices/ characteristics-fair-vaccine-injury-compensation-prog ram-for-canada

22. Ikeda SI, Hineno A, Ozawa K, Kinoshita T. Suspected adverse effects after human papillomavirus vaccination: a temporal relationship. Immunol Res 2018;66(6):723-5. DOI PubMed
23. Baumann A, Andersen B, Østergaard L, Larsen MB. Sense \& sensibility: decision-making and sources of information in mothers who decline HPV vaccination of their adolescent daughters. Vaccine X 2019;2:100020. DOI PubMed

24. Système canadien de surveillance des effets secondaires suivant l'immunisation. Ottawa (ON) : Gouvernement du Canada; (modifié 2019-12-11; accédé 2021-03-31). https:// www.canada.ca/fr/sante-publique/services/immunisation/ systeme-canadien-surveillance-effets-secondaires-suivantimmunisation.html

25. World Health Organization. Vaccination and trust: how concerns arise and the role of communication in mitigating crises. Copenhagen (DK): WHO; 2017. https://www.euro. who.int/_data/assets/pdf_file/0004/329647/Vaccines-andtrust.PDF

26. Kottasova I, Dean S, Sealy A. Norway reviewing deaths of frail and elderly patients vaccinated against COVID-19. Toronto (ON): CTV News; 2021-01-19 (modifié 2021-01-19; accédé 2021-03-31). https://www.ctvnews.ca/health/ coronavirus/norway-reviewing-deaths-of-frail-and-eld erly-patients-vaccinated-against-covid-19-1.5272547

27. Vaudry W, Zhao L, Stirling R; au nom du Comité consultatif national de l'immunisation (CCNI). Résumé de la Déclaration sur la vaccination antigrippale saisonnière pour la saison 2018-2019 du Comité consultatif national de l'immunisation (CCNI). Relevé des maladies transmissibles au Canada 2018;44(6):138-44. DOI

28. Major D, Cullen C. Canada shouldn't take vaccine doses from COVAX partnership, says Canada's former UN envoy. CBC News; (modifié 2021-02-26; accédé 2021-04-18). https://www.cbc.ca/news/politics/canada-stephe n-lewis-covax-1.5930344

29. Association médicale canadienne. Code d'éthique et de professionnalisme de I'AMC. Ottawa (ON) : AMC; 2018. https://www.cma.ca/fr/code-dethique-et-d e-professionnalisme-de-lamc

30. Canadian Nurses Association. Code of ethics for registered nurses, 2017 edition. Ottawa (ON): CNA; 2017. https://www.cna-aiic.ca/ /media/cna/page-content/pdf-en/ code-of-ethics-2017-edition-secure-interactive 